\title{
Organization Communication Factors and Abnormal Mammogram Follow-up: a Qualitative Study Among Ethnically Diverse Women Across Three Healthcare Systems
}

\author{
Jazmine D. Kenny, $M S P H^{7}$, Leah S. Karliner, MD MAS ${ }^{2}$, Karla Kerlikowske, $M D^{3}$, \\ Celia P. Kaplan, DrPH' , Ana Fernandez-Lamothe, BA², and Nancy J. Burke, PhD ${ }^{7}$
}

'Department of Public Health, School of Social Sciences, Humanities, and Arts, University of California Merced, CA, USA; ${ }^{2}$ Division of General Internal Medicine, Department of Medicine at the University of California San FranciscoSan Francisco, CA, USA; ${ }^{3}$ Department of Medicine and Epidemiology/Biostatistics, University of California San FranciscoSan Francisco, CA, USA.

\begin{abstract}
BACKGROUND: Regular mammogram screening for eligible average risk women has been associated with early detection and reduction of cancer morbidity and mortality. Delayed follow-up and resolution of abnormal mammograms limit early detection efforts and can cause psychological distress and anxiety.

OBJECTIVE: The goal of this study was to gain insight from women's narratives into how organizational factors related to communication and coordination of care facilitate or hinder timely follow-up for abnormal mammogram results.

DESIGN: We conducted 61 qualitative in-person interviews with women from four race-ethnic groups (African American, Chinese, Latina, and White) in three different healthcare settings (academic, community, and safety-net). PARTICIPANTS: Eligible participants had an abnormal mammogram result requiring breast biopsy documented in the San Francisco Mammography Registry in the previous year.
\end{abstract}

APPROACH: Interview narratives included reflections on experience and suggested improvements to communication and follow-up processes. A grounded theory approach was used to identify themes across interviews.

KEY RESULTS: Participants' experiences of follow-up and diagnosis depended largely on communication processes. Twenty-one participants experienced a follow-up delay ( $>$ 30 days between index mammogram and biopsy). Organizational factors, which varied across different institutions, played key roles in effective communication which included (a) direct verbal communication with the ability to ask questions, (b) explanation of medical processes and terminology avoiding jargon, and (c) use of interpretation services for women with limited English proficiency.

CONCLUSION: Health organizations varied in their processes for abnormal results communication and availability of support staff and interpretation services. Women who received care from institutions with more robust support staff, such as bilingual navigators, more often than not reported understanding their results and timely abnormal mammogram follow-up. These reports were consistent across women from diverse ethnic groups and

Received January 7, 2020

Accepted June 5, 2020

Published online June 29, 2020 suggest the value of organizational support services between an abnormal mammogram and resolution for improving follow-up times and minimizing patient distress.

KEY WORDS: abnormal mammogram; facility communication; delay.

J Gen Intern Med 35(10):3000-6

DOI: $10.1007 / \mathrm{s} 11606-020-05972-2$

(C) Society of General Internal Medicine 2020

\section{INTRODUCTION}

Regular mammogram screening for eligible average risk women has been associated with early detection and reduction of cancer morbidity and mortality. ${ }^{1}$ Delayed abnormal mammogram follow-up and resolution limit early detection efforts and can cause psychological distress and anxiety. ${ }^{2-5}$ Differences in breast cancer incidence, abnormal mammogram results follow-up, and time to diagnosis are more likely among ethnic minority groups such as African Americans, Asian-Americans, and Latinas. ${ }^{6-9}$ African American and Latinx women experience lower diseasespecific survival rates, despite non-Hispanic White women having an increased risk of developing breast cancer. ${ }^{10}$ Other identified risk factors for delayed follow-up and resolution include belonging to an ethnic minority group, having low income, and experiencing perceived discrimination. ${ }^{11}$

Much of the research on abnormal mammogram follow-up delays focuses on individual risk factors with limited consideration of organizational or communication factors. Yet some evidence suggests that organizational factors play an important role in timely follow-up. Studies have identified variation across facilities and found that facilities serving vulnerable populations had lower rates of biopsy and longer follow-up times. ${ }^{12,13}$ Furthermore, non-English speakers have additional barriers navigating and accessing care. ${ }^{4,14-17}$

Communication among referring physicians, mammography facilities, and patients also presents challenges. Previous research has identified patient difficulty comprehending mammogram results. ${ }^{11,18}$ Patients notified only by the federally mandated letter are less likely to be aware of their abnormal 
result, and more likely to have delayed follow-up compared to those with provider communication in person or over the telephone. ${ }^{19}$ Patients in facilities that used electronic medical records to notify patients for follow-up were also more likely to experience delay compared with those who received results via telephone. ${ }^{20}$

Interventions to address communication barriers will need to focus on providers and healthcare facilities to improve follow-up rates. ${ }^{21}$ Recommendations for abnormal test result communication include clearly defining timely follow-up, outlining provider responsibility to communicate results, having fail-safe procedures to ensure delivery, and specifying preferred mechanism of patient notification for critical tests. ${ }^{22}$ Effective healthcare system interventions require understanding of multilevel influences contributing to timely or delayed follow-up through attention to individual patient experience, including patient education and support, and changes to the delivery and information systems (i.e., navigation, patient tracking, and physician reminders). ${ }^{23}$

The goal of this study was to explore abnormal mammogram follow-up experiences reported in in-depth qualitative interviews with an ethnically diverse sample of participants (African American, Chinese, Latina, and White) receiving care in different healthcare settings (academic, community, and safety-net). Our analysis highlights communication practices and institutional arrangements that both supported and hindered timely follow-up.

\section{METHODS}

Participants were recruited through the San Francisco Mammography Registry (SFMR), part of the National Cancer Institute's Breast Cancer Surveillance Consortium (http:// breastscreening.cancer.gov). ${ }^{24}$ Recruitment was rolling with continuous monitoring to balance participants across each race/ethnicity (African American, Chinese, Latina, White) and across three health settings. Study eligibility criteria included the following: (1) having an abnormal mammogram (breast imaging reporting data system-BIRADS assessment -4 or 5) with a recommendation for biopsy in the prior year at one of three SFMR participating health organizations (academic, community, safety-net); (2) did not decline to be contacted for further research on the SFMR questionnaire; (3) speaks English, Spanish, or Cantonese; (4) ages 40 to 74; and (5) no history of ductal carcinoma in situ (DCIS) or invasive breast cancer prior to the index abnormal mammogram. The study was approved by the UCSF Committee on Human Research.

All three healthcare facilities serve a diverse urban population. The safety-net facility serves primarily publicly insured and uninsured patients, including a high proportion (approximately one third) of patients with limited English proficiency (LEP). The largest non-English language groups served are Spanish- and Chinese-speaking. This safety-net facility is home to a fully integrated cancer navigation program that provides services in multiple languages. The academic facility serves a primarily insured population, including those publicly insured, and approximately 10\% with LEP. Primary nonEnglish language groups served are Spanish and Cantonese. The community facility serves a diverse insured population, with the smallest proportion of patients with LEP of the three sites. This site has a large ethnically Chinese population and the primary non-English language group served is Cantonese. All three sites report having available professional in-person interpretation, by telephone and videoconferencing.

We defined delay as follow-up that occurred more than 30 days after the abnormal result. The 30-day cutoff for biopsy after a BIRADS $4 / 5$ result has both clinical relevance and face validity based on recommendations for rapid communication and biopsy follow-up of results which are suspicious for (BIRADS 4) or highly suggestive of (BIRADS 5) cancer. ${ }^{25}$

Eligible participants were mailed an invitation letter with an opt-out postcard. Two weeks later, research assistants called women who did not opt out to confirm eligibility and schedule in-person interviews. From the 254 women initially identified in the SFMR as potentially eligible, 25 were ultimately ineligible (cancer diagnosis, deceased); we were unable to reach 79 , and 14 were not contacted due to target interview numbers already achieved from that site (safety-net facility). Of the remaining 136, 15 opted out via postcard, 53 refused participation, 7 no-showed for a scheduled visit, and 61 completed interviews for a participation rate of $45 \%$.

Interviews were conducted by one of three female interviewers (Latinx, Chinese, and African American). Each interviewer was trained by the senior author, a medical anthropologist. None of the interviewers were previously known to any of the participants. Participants signed consent, were interviewed in their preferred language, and completed a brief demographic survey. In-depth semi-structured interviews lasted 40-60 minutes. Participants were asked to reflect on their care experience at various points in time since their index mammogram, including how they were first notified of their results, who they interacted with, and how they felt about the interaction. Participants were asked to recall experiences scheduling appointments, subsequent procedures, coordination of follow-up care, and whether they had specific recommendations for improvements. Interviews were audiotaped, de-identified, translated into English, and transcribed verbatim. Each participant was reimbursed for transportation/parking and given $\$ 40$ in appreciation of her time. If women had specific health concerns or medical questions, they were encouraged to contact their primary care provider. A study physician was available by phone to speak with participants as needed.

Interview transcripts were uploaded into ATLAS.ti version 7 for analysis. ${ }^{26}$ Using an approach of open coding informed by grounded theory, ${ }^{27}$ the first author (JK) coded each transcript in consultation with the senior author (NB). Inductive codes were developed from concepts that emerged from women's reported experiences. Throughout the coding process, two team members (JK and NB) met to discuss codes and 
emergent themes. Once all transcripts were coded, we conducted "co-occurrences" to evaluate associations between specific codes and identify patterns in the data. We created analytically appropriate code groups, ran queries, and drafted theoretical memos reporting patterns and themes that emerged from the data. These patterns became the themes reported in our findings.

\section{RESULTS}

Of the 61 women interviewed, $40(66 \%)$ were classified as having timely follow-up (13 Spanish-speakers, 24 English, 3 Cantonese) and 21 (34\%) as delayed follow-up (4 Spanishspeakers, 15 English, 2 Cantonese). Participant descriptive characteristics by follow-up status are presented in Table 1. Half of the participants received care in the safety-net setting $(n=30)$. Table 2 shows participants by race/ethnicity and healthcare facility.

In the following, we report women's experiences learning of their mammography results and subsequent follow-up communication. Experiences reported ranged from those with one mammogram and subsequent diagnostic follow-up to those who underwent multiple mammograms, biopsies, and clinic visits. Therefore, findings reported reflect multiple time points. All participant's names are pseudonyms.

\section{Unidirectional vs. Bidirectional Communication}

Mammography results communication varied both across and within facilities. We classified results delivered via letter/mail, electronic medical record, or voicemail as unidirectional since women could not readily engage with a live person to ask questions. Interactive bidirectional communication created opportunities to ask questions and cultivate engagement. Women described one-way communication through voice messages as limiting their understanding of next steps and connection to the clinic. Those who received unclear information expressed frustration and confusion, and, in some cases, this led to delayed follow-up.

Table 1 Descriptive Characteristics of Study Participants by (1) Timely and (2) Delayed Follow-up

\begin{tabular}{lll}
\hline \hline & Timely $\boldsymbol{N ( \% )}$ & Delayed $\boldsymbol{N ( \% )}$ \\
\hline Facility & & \\
$\quad$ Safety-net & $22(55)$ & $8(38)$ \\
Academic & $14(35)$ & $10(48)$ \\
Community & $4(10)$ & $3(14)$ \\
Language & & \\
English & $24(60)$ & $15(71)$ \\
Spanish & $13(33)$ & $4(19)$ \\
Cantonese & $3(7)$ & $2(10)$ \\
Race/Ethnicity & $14(35)$ & $5(24)$ \\
Latina & $9(22.5)$ & $6(28)$ \\
White & $9(22.5)$ & $5(24)$ \\
African American & $8(20)$ & $5(24)$ \\
Chinese & $40(66)$ & $21(34)$ \\
Total N & &
\end{tabular}

Table 2 Descriptive Table of Study Participants by Facility and Race/Ethnicity

\begin{tabular}{|c|c|c|c|c|}
\hline $\begin{array}{l}\text { Healthcare } \\
\text { facility by } \\
\text { participant } \\
\text { race/ethnicity }\end{array}$ & $\begin{array}{l}\text { Academic } \\
\text { facility }\end{array}$ & $\begin{array}{l}\text { Community } \\
\text { facility }\end{array}$ & $\begin{array}{l}\text { Safety- } \\
\text { net } \\
\text { facility }\end{array}$ & Totals \\
\hline $\begin{array}{l}\text { African } \\
\text { American }\end{array}$ & $6(25 \%)$ & $2(29 \%)$ & \multirow{5}{*}{$\begin{array}{l}6 \\
(20 \%) \\
16 \\
(53 \%) \\
4 \\
(13 \%) \\
4 \\
(13 \%) \\
30 \\
(49 \%)\end{array}$} & \multirow{5}{*}{$\begin{array}{l}14 \\
(23 \%) \\
19 \\
(31 \%) \\
15 \\
(25 \%) \\
13 \\
(21 \%) \\
61 \\
(100 \%)\end{array}$} \\
\hline Latina & $2(8 \%)$ & $1(14 \%)$ & & \\
\hline White & $8(33 \%)$ & $3(43 \%)$ & & \\
\hline Chinese & $8(33 \%)$ & $1(14 \%)$ & & \\
\hline Totals & $24(39 \%)$ & $7(12 \%)$ & & \\
\hline
\end{tabular}

For example, the academic facility left Bonnie a voice message regarding her index mammogram results which she reported left her feeling "frantic" not knowing what to do next. The message included an incorrect follow-up phone number and no information on who to contact.

They left a message and they weren't very informative. I wasn't very impressed with the way they presented the information...my mammogram was abnormal but they couldn't give me any information. That was frustrating too, why it wasn't normal.

(English-speaking, White, Delayed, Academic Facility)

Patient-initiated tasks such as calling the healthcare facility for information and making appointments were sometimes perceived as difficult due to limited information or language barriers. Josefina expressed her frustration trying to make appointments over the phone:

To schedule an appointment is very complicated. I almost always go in person because I don't understand them...One person would transfer me to another, the other to another one...I was so frustrated.

(Spanish-speaking, Latina, Timely, Safety-net Facility)

Women reported that receiving mammography results through voice message or mailed letter with general language impeded on their ability to follow-up.

On the other hand, women who experienced bidirectional communication with facility staff reported positive experiences. Faith was notified of her mammogram results via telephone and the clinic immediately scheduled in-person meetings with her providers.

[The clinic] called and they made an appointment for me to come back. I actually had to go talk to the doctors first because they'd determined that I needed the [biopsy]... They wanted to know how my mindset was... trying to put me at ease...trying to relieve my anxiety. 
(English-speaking, African American, Timely, Safetynet Facility)

Two weeks following a second mammogram, Barbara was given her results, including the need for a follow-up biopsy, at the community facility. Since it was the first time Barbara had a biopsy, she explained, “I didn't really know what to expect, and I didn't know what to compare it to... [but] they did a good job of explaining it." In addition to talking with a staff member in person, Barbara was given a pamphlet on the procedure, which she reported helped her understand what to expect.

Joanna, who lost her husband to cancer, reported feeling scared about being referred to the cancer clinic, but overall had positive interactions with her $\mathrm{PCP}$ and the academic facility staff throughout her two mammograms, ultrasound, and biopsy. A staff member from the academic facility's cancer center explained she would receive the biopsy results from her PCP. Before she made the call, a staff member from the PCP's office called to schedule an appointment less than a week later. Her PCP explained the biopsy results and scheduled follow-up appointments every six months. Joanna explained that the support of her daughters, prayer, and her PCP helped her feel reassured. She also noted PCP office staff and the academic facility were available to answer questions over the phone and in person.

Women reported bidirectional communication of follow-up with providers or supplemental personnel was helpful in understanding next steps, since these forms of communication provided the opportunity to clarify information, make appointments, and receive support.

\section{Complex Processes and Medical Jargon}

In the following, we highlight participants' perceptions of provider explanations, and how this affected participant comprehension of diagnoses, next steps, and/or treatment. Women reported difficulty understanding providers who used medical jargon, complicated explanations of procedures, or provided results without context. For example, Mona, who previously discussed the possibility of extracting her mammary glands with a genetic advisor due to a family history of cancer, reported that she was not given information beyond her biopsy results despite wanting an explanation of what was extracted.

[The facility] only sent me a letter explaining that [the mass] was big but it was okay. Basically, the only thing that they specified was that it wasn't cancerous. They never explained to me why it happened, why it grew, whether there was a possibility for it to grow back in the same place or if anything was extracted; I never knew. I didn't even know who to ask about those things. (Spanish-speaking, Latina, Delayed, Safety-net Facility)

Maya reported not understanding the medical terminology in her letter,
Doctors have big words and you don't understand... Just break it down to me...A lot of doctors' terms are not something I would just know...I think we as patients need to be responsible and clarify whatever you don't know because at the end of the day it's about you. (English-speaking, African American, Timely, Safetynet Facility)

Several women expressed confusion regarding the breast tissue marker, or tag, inserted after the biopsy. In general, this tissue marker is placed at the biopsy site for subsequent breast examinations and future provider reference. Although many of the participants who mentioned the tag were not considered delayed by our definition, they described their experiences as negative since they did not fully understand the procedures. As Martha noted,

They just told me they were going to implant the microchip... They didn't tell me the side effects. I would like them to be more honest and more open, so the patient can decide. 'I am going to do it knowing the risks, but I am aware of what I am going to do.' (Spanish-speaking, Latina, Timely, Safety-net Facility)

Daniela's most recent mammogram resulted in an abnormal finding, and her provider explained she needed to choose between a biopsy or wait until her next routine mammogram for follow-up. Although Daniela had had a biopsy in the past, she reported feeling scared making this decision on her own.

I panicked. Another biopsy...There is the option that if it does not grow it can be there, but it was like- - I did not know what to do. I needed someone to tell me, 'You have to do this because it is the best option for you.'

(Spanish-speaking, Latina, Delayed, Academic Facility)

Another participant reported wanting her results via letter or online portal because she had difficulty understanding medical terms over the phone. Mina explained,

If the results are in hard copy, at least I read it...as opposed to someone calling me and trying to take notes...[A staff member] read [the results], and like I said, it's very hard to try to understand a medical term, right? I got stuck with how to even spell it...[It's] a moot point because I can't retain what he just said. (Chinese, English-speaking, Timely, Community Facility)

Participants described instances in which medical terminology and processes were clearly explained. Healthcare personnel played an important role in such communications by helping women follow recommended timelines 
and putting them at ease. For example, Jane described:

The pathologist...tells [other providers] in their language what's going on. Then, translated it to me in layman's terms. Then, they...tell us right away if there's cancer or anything like that...It hasn't been so far...they're on top of it.

(English-speaking, African American, Timely, Academic Facility)

Similarly, Lupe reported how her patient navigator helped her decide to schedule a biopsy:

My navigator [explained] the process and gave me support... In the midst of all the worry and the sadness that you feel, at the same time you also feel joy from knowing that there are people who care about what's happening to you. [They're] trying to make you feel more confident about making decisions. In this case, mine was to get [a biopsy] sooner.

(Spanish-speaking, Latina, Timely, Safety-net Facility)

When asked about recommendations for other women going through a similar experience, several participants mentioned having an advocate figure to help patients. For example, Fei highlighted her positive experience with a nurse who also served as her interpreter,

Although the doctors speak English, they have translators for you, especially the nurse. She can explain things well and she's a caring person. She knows how to [explain] diplomatically, which is really comforting... She makes you relaxed by telling you there's nothing to worry about...The way one talks to you does make a difference. (Cantonese-speaking, Chinese, Timely, Safety-net Facility)

Women reported feeling supported during engaging interactions with knowledgeable healthcare staff, including patient navigators and nurses.

In addition to the complexity of medical jargon, the availability of interpretation services - such as those provided by Fei's nurse - contributed to varying experiences for women during their mammogram follow-up process. At the same time, the unavailability of these services undermined participants' understanding of complex medical processes and jargon. Following two mammograms, Carmen's provider from the safety-net facility asked if she wanted a biopsy or an ultrasound; however, these options were communicated in English while Carmen's primary language was Spanish. No interpreter was present during this interaction. Carmen reported feeling unsure about her understanding of what she was being asked to choose. She left this interaction angry and ultimately experienced a delay in follow-up. Similarly, in response to a question about her overall experience interacting with her provider, Rosa, also Spanish-speaking, mentioned that the follow-up process at the safety-net facility should have been faster. When asked about her most recent appointment, she responded, "I thought, 'If I ask for an interpreter, it's going to take longer and I want to go.' So I said, 'No, it's fine."' Rosa ended up experiencing a delayed follow-up.

LEP participants reported that the absence of professional interpreters or bilingual staff impeded their abilities to fully engage in the follow-up process. In contrast, participants reported that healthcare facilities with language concordant staff-in the form of providers, social workers, or navigators - contributed to positive experiences and often led to timely follow-up. This was particularly the case for patients from the safety-net facility, which serves the largest proportion of LEP patients, provides in-person and telephone interpretation, and has a fully integrated cancer navigation program.

Bella reported feeling scared about her abnormal results and upcoming biopsy. A bilingual social worker assisted her with paperwork, set up appointments, and was present before the biopsy procedure. Bella explained, "[The social worker] was like my guardian angel and never left me. Every time I had a doubt, I would tell her...I looked for comfort in the social worker... she transmitted a lot of confidence to me." (Spanish-speaking, Latina, Timely, Safety-net Facility)

Juanita described a similar experience, in which a Spanishspeaking patient navigator served as a translator and a support.

The [patient navigator] was holding my hand the whole time, telling me everything was fine...The doctor called me to say I shouldn't worry, because everything was fine. They would send me a follow-up appointment, and if I wanted to come to have things explained in person, I could... [The doctor] called me in English, but the girl who spoke Spanish was right there... I felt better. I felt happier.

(Spanish-speaking, Latina, Timely, Safety-net Facility)

Lan recalled how her follow-up was explained to her:

I asked [the staff] if there was anyone speaking in Cantonese...they had an interpreter. The nurse would speak to the interpreter first, then the interpreter would translate for me in Cantonese... When I had my breast checked each time...I did not have to worry... because they had a Chinese interpreter for me.

(Cantonese-speaking, Chinese, Timely, Safety-net Facility)

Participants also noted provider linguistic ability as a strength. As Valentina stated, "[My doctor] understands a lot of Spanish...it wasn't very difficult for her to communicate and explain [the mammogram results] to me... She explained it 
to me properly in Spanish.” (Spanish-speaking, Latina, Timely, Safety-net Facility)

As these participants note, having a professional interpreter or language concordant staff positively impacted their experiences and sometimes alleviated distress.

\section{DISCUSSION}

Our study explored abnormal mammogram follow-up experiences of ethnically diverse women across three healthcare organizations. Participants' experiences depended largely on organizational processes and provider communication, suggesting facilities with support mechanisms and bilingual personnel (i.e., patient navigators, clinicians, nurses, professional interpreters, social workers) who provide explanations in lay language were more likely to facilitate timely and functional follow-up. These services were present in all three facilities to some degree, but utilization varied across and within facilities. Participants described differences in how information was presented, how clearly complex medical processes and terminology were explained, and whether interpretation services were provided.

Zapka and colleagues conducted a systematic review assessing factors that contributed to quality care during abnormal screening follow-up and developed an ecological model to contextualize these factors. ${ }^{23}$ Our participants identified several organizational factors present in Zapka's ecological model that either facilitated (e.g., patient navigators) or undermined (e.g., organizational challenges) timely follow-up.

Our findings are consistent with others who reported dissatisfaction with results communication, including difficulty comprehending the notification letter, preference for in-person communication, supplemental print material, and an action plan/hotline to ask questions. ${ }^{18,28}$ Since mammography result notification letters are often written at a high reading level, they may add to confusion rather than help. ${ }^{29}$ A systematic review of various communication methods in cancer screening programs, including mammography, found speed, accuracy of results, visual support, and ability to ask questions were preferred by participants. ${ }^{30}$

Several studies have reported the influence of patient navigators in alleviating cancer care delays due to their patientcentered approaches to care complexities. ${ }^{27-34}$ Patient navigation has been successful in gaining appropriate follow-up and shortening time following abnormal mammograms. 3, 32, 35, 36 Although patient navigation programs are a strategy to address mammogram process barriers, particularly for vulnerable women, ${ }^{35-40}$ these programs can be costly and require additional trained personnel. While the safety-net facility was the only facility in our study that had an established patient navigator program, some women in the safety-net facility still experienced delays to follow-up. Previous research conducted by members of our team found that facilities that serve vulnerable women have longer follow-up times. ${ }^{13}$
Our findings suggest clear, lay language communicated through existing healthcare facility personnel may contribute to increased timely follow-up. Healthcare providers who engage directly with their patients about health experiences improve patient experience. ${ }^{3,41}$ Our study found that this positive effect may be moderated among LEP patients by provider use of complex medical terminology and instruction, which may limit patient engagement and contribute to a poorer experience. Participants reported the value of discussing mammogram results with healthcare facility staff who were able to contextualize complex medical terminology in the participant's preferred language. Poor communication could be connected to limitations in facility staff capacity and unclear definitions of timely follow-up. ${ }^{13,22,23}$ Our findings, consistent with previous research, ${ }^{39,42}$ highlight the importance of health systems providing LEP patients with access to interpretation services. Some participants, for example, reported feeling confident making decisions when ancillary staff or a provider could explain results and next steps in the same language.

Our study highlights key factors in effective facility/ provider communication with patients receiving abnormal mammogram results including the following: (a) bidirectional communication with the ability to ask questions, (b) explanation of medical processes and terminology in lay language, (c) provision of navigational assistance for those who need it, and (d) use of interpretation services or knowledgeable language concordant staff for communication with women with LEP. Explanation of medical terminology and processes in women's preferred language through bidirectional communication with healthcare personnel may assist with patient comprehension of abnormal results, and minimize delays to resolution and psychological distress among vulnerable women.

Acknowledgments: The authors wish to express their gratitude to the project interviewers, the study participants, partnering agencies, and other project staff (Sarita Pathak) for their contributions in study implementation and data collection.

Corresponding Author: Nancy J. Burke, PhD; Department of Public Health, School of Social Sciences, Humanities, and Arts, University of California Merced, CA, USA (e-mail: nburke2@ucmerced.edu).

Funding Information This research was supported by the National Institutes of Health Exploratory/Developmental Research Grant [R21CA195429] and the National Institutes of Health National Cancer Institute Research Program Project Grant [P01CA154292].

\section{Compliance with Ethical Standards:}

Conflict of Interest: The authors declare that they do not have a conflict of interest.

\section{REFERENCES}

1. Oeffinger KC, Fontham ETH, Etzioni R, et al. Breast Cancer Screening for Women at Average Risk: 2015 Guideline Update From the American Cancer Society. JAMA. 2015;314(15):1599-1614 
2. Brett J, Bankhead C, Henderson B, Watson E, Austoker J. The psychological impact of mammographic screening. A systematic review. Psychooncology. 2005; 14(11):917-938.

3. Molina Y, Beresford SAA, Constant TH, Thompson B. Conversations about Abnormal Mammograms on Distress and Timely Follow-up Across Ethnicity. J Cancer Educ. 2017;32(2):320-327.

4. Molina Y, Beresford SAA, Espinoza N, Thompson B. Psychological Distress, Social Withdrawal, and Coping Following Receipt of an Abnormal Mammogram Among Different Ethnicities: A Mediation Model. Oncol Nurs Forum. 2014;41(5):523-532.

5. Tosteson ANA, Fryback DG, Hammond CS, et al. Consequences of False-Positive Screening Mammograms. JAMA Intern Med. 2014;174(6):954-961.

6. Ferrante JM, Chen P-H, Kim S. The Effect of Patient Navigation on Time to Diagnosis, Anxiety, and Satisfaction in Urban Minority Women with Abnormal Mammograms: A Randomized Controlled Trial. J Urban Health. 2008;85(1):114-124.

7. Nguyen KH, Pasick RJ, Stewart SL, Kerlikowske K, Karliner LS. Disparities in abnormal mammogram follow-up time for Asian women compared with non-Hispanic white women and between Asian ethnic groups. Cancer. 2017;123(18):3468-3475.

8. SEER. Annual Report to the Nation 2017: Incidence Highlights. https:// seer.cancer.gov/report_to_nation/incidence.html. Published 2018. Accessed March 21, 2018.

9. Warner ET, Tamimi RM, Hughes ME, et al. Time to diagnosis and breast cancer stage by race/ethnicity. Breast Cancer Res Treat. 2012;136(3):813-821.

10. American Cancer Society. Breast Cancer Facts and Figures 2017-2018. American Cancer Society; 2017.

11. Karliner LS, Patricia Kaplan C, Juarbe T, Pasick R, Pérez-Stable EJ. Poor Patient Comprehension of Abnormal Mammography Results. J Gen Intern Med. 2005;20(5):432-437.

12. Goldman LE, Walker R, Hubbard R, Kerlikowske K. Breast Cancer Surveillance Consortium. Timeliness of abnormal screening and diagnostic mammography follow-up at facilities serving vulnerable women. Med Care. 2013;51(4):307-314

13. Karliner LS, Kaplan C, Livaudais-Toman J, Kerlikowske K. Mammography facilities serving vulnerable women have longer follow-up times. Health Serv Res. 2019;54 Suppl 1:226-233.

14. Fernandez A, Schillinger D, Warton EM, et al. Language Barriers, Physician-Patient Language Concordance, and Glycemic Control Among Insured Latinos with Diabetes: The Diabetes Study of Northern California (DISTANCE). J Gen Intern Med. 2011;26(2):170-176.

15. Karliner L, Ma L, Hofmann M, Kerlikowske $\mathbf{K}$. Language Barriers, Location of Care and Delays in Follow-up of Abnormal Mammograms. Med Care. 2012;50(2):171-178.

16. Schwei RJ, Pozo SD, Agger-Gupta N, et al. Changes in research on language barriers in health care since 2003: A cross-sectional review study. Int J Nurs Stud. 2016;54:36-44.

17. Terui S. Conceptualizing the Pathways and Processes Between Language Barriers and Health Disparities: Review, Synthesis, and Extension. J Immigr Minor Health. 2017;19(1):215-224.

18. Marcus EN, Drummond D, Dietz N. Urban Women's Preferences for Learning of Their Mammogram Result: A Qualitative Study. J Cancer Educ. 2012;27(1):156-164.

19. Pérez-Stable EJ, Afable-Munsuz A, Kaplan CP, et al. Factors influencing time to diagnosis after abnormal mammography in diverse women. $\mathrm{J}$ Womens Health 2002. 2013;22(2):159-166.

20. Schapira MM, Barlow WE, Conant EF, et al. Communication Practices of Mammography Facilities and Timely Follow-up of a Screening Mammogram with a BI-RADS 0 Assessment. Acad Radiol. 2018.

21. Felix HC, Bronstein J, Bursac Z, Stewart MK, Foushee HR, Klapow J. Family Planning Provider Referral, Facilitation Behavior, and Patient Follow-up for Abnormal Pap Smears. Public Health Rep. 2009; 124(5):733-744.

22. Singh H, Vij MS. Eight Recommendations for Policies for Communicating Abnormal Test Results. Jt Comm J Qual Patient Saf. 2010;36(5):226-AP2.
23. Zapka J, Taplin SH, Anhang Price R, Cranos C, Yabroff R. Factors in Quality Care-The Case of Follow-Up to Abnormal Cancer Screening Tests-Problems in the Steps and Interfaces of Care. JNCI Monogr. 2010;2010(40):58-71.

24. Ballard-Barbash R, Taplin SH, Yankaskas BC, et al. Breast Cancer Surveillance Consortium: a national mammography screening and outcomes database. AJR Am J Roentgenol. 1997;169(4): 1001-1008.

25. Eberl MM, Fox CH, Edge SB, Carter CA, Mahoney MC. BI-RADS Classification for Management of Abnormal Mammograms. J Am Board Fam Med. 2006;19(2):161-164.

26. ATLAS.Ti. Berlin, Germany; 2013.

27. Charmaz K. Constructing Grounded Theory. 2nd ed. Sage; 2014.

28. Allen J, Shelton R, Harden E, Goldman R. Follow-up of abnormal screening mammograms among low-income ethnically diverse women: Findings from a qualitative study. Patient Educ Couns. 2008;72(2):283292.

29. Marcus EN, Sanders LM, Pereyra M, et al. Mammography Result Notification Letters: Are They Easy to Read and Understand? J Womens Health. 2011;20(4):545-551.

30. Williamson S, Patterson J, Crosby R, et al. Communication of cancer screening results by letter, telephone or in person: A mixed methods systematic review of the effect on attendee anxiety, understanding and preferences. Prev Med Rep. 2019;13:189-195.

31. Ali-Faisal SF, Colella TJF, Medina-Jaudes N, Benz Scott L. The effectiveness of patient navigation to improve healthcare utilization outcomes: A meta-analysis of randomized controlled trials. Patient Educ Couns. 2017;100(3):436-448.

32. Freeman HP. The History, Principles, and Future of Patient Navigation: Commentary. Semin Oncol Nurs. 2013;29(2):72-75.

33. Glassgow AE, Molina Y, Kim S, Campbell RT, Darnell J, Calhoun EA. A Comparison of Different Intensities of Patient Navigation After Abnormal Mammography. Health Promot Pract. 2018;1524839918782168.

34. Hoffman HJ, LaVerda NL, Young HA, et al. Patient Navigation Significantly Reduces Delays in Breast Cancer Diagnosis in the District of Columbia. Cancer Epidemiol Prev Biomark. 2012;21(10):1655-1663.

35. Ell K, Vourlekis B, Lee P-J, Xie B. Patient navigation and case management following an abnormal mammogram: A randomized clinical trial. Prev Med. 2007;44(1):26-33.

36. Gabitova G, Burke NJ. Improving healthcare empowerment through breast cancer patient navigation: a mixed methods evaluation in a safetynet setting. BMC Health Serv Res. 2014;14:407.

37. Molina Y, Hempstead BH, Thompson-Dodd J, et al. Medical Advocacy and Supportive Environments for African-Americans Following Abnormal Mammograms. J Cancer Educ Off $\mathrm{J}$ Am Assoc Cancer Educ. 2015;30(3):447-452.

38. Dudley DJ, Drake J, Quinlan J, et al. Beneficial Effects of a Combined Navigator/Promotora Approach for Hispanic Women Diagnosed with Breast Abnormalities. Cancer Epidemiol Prev Biomark. 2012;21(10): 1639-1644.

39. Markossian TW, Darnell JS, Calhoun EA. Follow-Up and Timeliness After an Abnormal Cancer Screening Among Underserved, Urban Women in a Patient Navigation Program. Cancer Epidemiol Prev Biomark. 2012;21(10): 1691-1700.

40. Percac-Lima S, Ashburner JM, McCarthy AM, Piawah S, Atlas SJ. Patient navigation to improve follow-up of abnormal mammograms among disadvantaged women. J Womens Health 2002. 2015;24(2):138-143.

41. Ziebland S, Coulter A, Calabrese JD, Locock L. Understanding and Using Health Experiences: Improving Patient Care. OUP Oxford; 2013.

42. Karliner LS, Pérez-stable EJ, Gregorich SE. Convenient Access to Professional Interpreters in the Hospital Decreases Readmission Rates and Estimated Hospital Expenditures for Patients With Limited English Proficiency. Med Care. 2017;55(3):199-206.

Publisher's Note: Springer Nature remains neutral with regard to jurisdictional claims in published maps and institutional affiliations. 